\title{
Functional Connectome of the Fetal Brain
}

\author{
DElise Turk, ${ }^{1,2}$ Marion I. van den Heuvel, ${ }^{3}$ Manon J. Benders, ${ }^{1,2}$ Roel de Heus, ${ }^{1}$ Arie Franx, ${ }^{1}$ Janessa H. Manning ${ }^{4,5}$ \\ (D)Jasmine L. Hect, ${ }^{4,5}$ Edgar Hernandez-Andrade, ${ }^{5,6}$ Sonia S. Hassan, 5,6,7 (D)Roberto Romero, 5,8,9,10 (D)René S. Kahn, ${ }^{11}$ \\ (D) Moriah E. Thomason, ${ }^{12,13,14 *}$ and (DMartijn P. van den Heuvel ${ }^{15,16 *}$ \\ ${ }^{1}$ Department of Neonatology and Obstetrics, University Medical Center Utrecht, ${ }^{2}$ UMC Utrecht Brain Center, Utrecht University, 3508 AB Utrecht, the \\ Netherlands, ${ }^{3}$ Department of Cognitive Neuropsychology, Tilburg University, 5000 LE Tilburg, The Netherlands, ${ }^{4}$ Merrill Palmer Skillman Institute for \\ Child and Family Development, Wayne State University, Detroit, Michigan 48202, ${ }^{5}$ Perinatology Research Branch, NICHD/NIH/DHHS, Bethesda, Maryland \\ and Detroit, Michigan, ${ }^{6}$ Departments of Obstetrics and Gynecology, ${ }^{7}$ Physiology, Wayne State University School of Medicine, Detroit, Michigan 48201, \\ ${ }^{8}$ Department of Obstetrics and Gynecology, University of Michigan, Ann Arbor, Michigan 48109, ${ }^{9}$ Department of Epidemiology and Biostatistics, Michigan \\ State University, East Lansing, Michigan 48824, ${ }^{10}$ Center for Molecular Medicine and Genetics, Wayne State University, Detroit, Michigan 48201, \\ ${ }^{11}$ Department of Psychiatry, Icahn School of Medicine at Mount Sinai, New York, New York 11029, ${ }^{12}$ Departments of Child and Adolescent Psychiatry, \\ ${ }^{13}$ Population Health, New York University Langone Health, New York, New York 10016, ${ }^{14}$ Neuroscience Institute, NYU Langone Health, New York, \\ ${ }^{15}$ Dutch Connectome Laboratory, Department of Complex Traits Genetics, Center for Neurogenomics and Cognitive Research, VU Amsterdam, 1081 HV \\ Amsterdam, The Netherlands, and ${ }^{16}$ Department of Clinical Genetics, VU University Medical Center, Amsterdam Neuroscience, 1081 HV Amsterdam, \\ The Netherlands
}

Large-scale functional connectome formation and reorganization is apparent in the second trimester of pregnancy, making it a crucial and vulnerable time window in connectome development. Here we identified which architectural principles of functional connectome organization are initiated before birth, and contrast those with topological characteristics observed in the mature adult brain. A sample of 105 pregnant women participated in human fetal resting-state fMRI studies (fetal gestational age between 20 and 40 weeks). Connectome analysis was used to analyze weighted network characteristics of fetal macroscale brain wiring. We identified efficient network attributes, common functional modules, and high overlap between the fetal and adult brain network. Our results indicate that key features of the functional connectome are present in the second and third trimesters of pregnancy. Understanding the organizational principles of fetal connectome organization may bring opportunities to develop markers for early detection of alterations of brain function.

Key words: brain development; fetal; functional connectivity; prenatal; resting-state fMRI

\section{Significance Statement}

The fetal to neonatal period is well known as a critical stage in brain development. Rapid neurodevelopmental processes establish key functional neural circuits of the human brain. Prenatal risk factors may interfere with early trajectories of connectome formation and thereby shape future health outcomes. Recent advances in MRI have made it possible to examine fetal brain functional connectivity. In this study, we evaluate the network topography of normative functional network development during connectome genesis in utero. Understanding the developmental trajectory of brain connectivity provides a basis for understanding how the prenatal period shapes future brain function and disease dysfunction.

\section{Introduction}

The brain is organized as a complex network of functionally communicating regions, a network also known as the functional con-

Received Nov. 7, 2018; revised Aug. 22, 2019; accepted 0ct. 1, 2019.

Author contributions: E.T., M.I.v.d.H., M.J.B., R.R., M.E.T., and M.P.v.d.H. designed research; E.T., M.J.B., R.d.H., A.F., R.S.K., M.E.T., and M.P.v.d.H. performed research; E.T., J.H.M., J.L.H., E.H.-A., S.S.H., and M.P.v.d.H. analyzed data; E.T., M.E.T., and M.P.v.d.H. wrote the paper.

This work was supported by awards to M.E.T. from the National Institutes of Health, MH110793, ES026022, ES020957, and by a National Alliance for Research on Schizophrenia and Depression Young Investigator Award; in part by the Perinatology Research Branch, Division of Obstetrics and Maternal-Fetal Medicine, Division of Intramural Research, Eunice Kennedy Shriver National Institute of Child Health and Human Development, National Institutes of Health, U.S. Department of Health and Human Services (NICHD/NIH/DHHS); and in part, with Federal funds from NICHD/NIH/DHHS under Contract HHSN275201300006C. Dr. Romero has contributed to this work as part of his nectome. The macroscale functional connectome is observable using MRI in infancy and studies have reported hallmark features, including the presence of functionally coupled modules, official duties as an employee of the U.S. Federal Government. M.P.H was sponsored by a fellowship of MQ by a VID Grant from the Netherlands Organization for Scientific Research (Grant 452-16-015). We thank Pavan Jella, Sophia Neuenfeldt, Toni Lewis, Bryan Turman, Tamara Qawasmeh, Joshua Hammond, and Sydney Brooks for their assistance in data acquisition and analyses, Lianne Scholtens and Siemon de Lange for their valuable contribution on the graphical content of the paper, and participant families who generously shared their time.

The authors declare no competing financial interests.

*M.E.T. and M.P.v.d.H. contributed equally as last authors.

Correspondence should be addressed to Elise Turk at E.turk-2@umcutrecht.nl.

https://doi.org/10.1523/JNEUROSCI.2891-18.2019

Copyright $\odot 2019$ the authors 
small world organization, and putative hubs that facilitate efficient global communication (Lin et al., 2008; Gao et al., 2009, 2015; Smyser et al., 2010; Ball et al., 2014; van den Heuvel et al., 2015). Functional connectome development of older children and adolescents has been characterized by a reconfiguration from a modular into a more global integrated network during adult life (Fair et al., 2009; Power et al., 2010). Examining the specific earlier age-stages of macroscale connectome maturation will provide important new insight into the architecture of human brain structure and function. In this paper, we will address important questions about the first phases after connectome genesis by evaluating the human fetal brain before birth.

Available evidence suggests that the macroscale connectome begins to take form when the majority of neurons have reached their final destination (Sidman and Rakic, 1973; Mrzljak et al., 1991; Huttenlocher and Dabholkar, 1997). Structural outgrowth is supported by spontaneous firing of neurons that reinforce appropriate connections and trigger essential activity-dependent signaling processes (Thomason, 2018) giving rise to structural and functional cortical connectivity (Kostović and JovanovMilošević, 2006; Vasung et al., 2010). This has led to the suggestion that basic principles of the functional connectome are initially established as early as during late second trimester of pregnancy (Collin and van den Heuvel, 2013; Hoff et al., 2013).

The trajectory of functional dynamics of brain development follows multiple stages, all influencing future connectome health. Prenatal processes such as maternal viral infections, extensive stress, food-intake, alcohol and medication use, among others, all have strong influence on later life functional connectivity (FC) of children (Grewen et al., 2015; Salzwedel et al., 2015, 2016; Li et al., 2016; Infante et al., 2017; van den Bergh et al., 2017; de Water et al., 2018; Rudolph et al., 2018). With advances in fetal restingstate neuroimaging it is now possible to measure these FC patterns noninvasively in vivo (for review, see Anderson and Thomason, 2013; van den Heuvel and Thomason, 2016). Mapping and understanding the healthy fetal functional connectome may bring opportunities for early detection of functional alterations of the vulnerable developing brain.

We understand relatively little about the onset of key principles of the functional connectome before birth. Researchers have begun to isolate foundational aspects of prenatal functional brain development, but these studies are strikingly few. Available studies show that fetal FC strength between homologous cortical regions increases with advancing age in a medial to lateral gradient (Thomason et al., 2013). Additionally, long-range functional connections, highly connected primary and association regions and first principals of resting-state networks as observed in (mostly preterm born) neonates have been detected across 20-40 weeks of gestational age (Schöpf et al., 2012, 2014; Jakab et al., 2014; Thomason et al., 2015; van den Heuvel et al., 2018). However, with the field of fetal FC rapidly growing, much remains to be understood about the onset and development of fundamental connectome properties during the early stages of connectome genesis.

The goal of the present study is to complement the field of fetal fMRI by using a large fetal resting-state fMRI dataset $(N=105)$ to evaluate the early presence of key characteristics of human connectome organization before birth. We aim to shed light on the blueprint of the connectome by comparing fetal topological characteristics to those observed in the mature adult brain. We apply graph analytical methods to better understand properties of small world organization, modularity, and rich-club hub or- ganization of functional brain dynamics during the late second and third trimester of pregnancy.

\section{Materials and Methods}

\section{Study population}

A total of 139 healthy, non-sedated pregnant women underwent MRI examination in their late second or third trimester of pregnancy. Singleton fetuses were scanned using a 3T MRI system at Wayne State University (van den Heuvel et al., 2018). Thirty-four of the 139 cases were excluded before analysis due to high movement or due to detectable health complications (e.g., subsequent preterm birth, preeclampsia), leaving the inclusion of 105 fetal subjects (39\% female, $61 \%$ male). Mean age of the fetuses at the time of MRI was 33.5 ( $S D=3.97$ ) weeks of gestation with a range of 20.6-39.6 weeks gestational age. Subjects were born, on average, at 39.3 weeks of gestation $(S D=1.22)$. Ethnic/racial distribution of the subjects was $81 \%$ African-American, $10.5 \%$ Caucasian, $2.9 \%$ bi-racial, and 5.7\% not disclosed. All procedures were performed as approved by the Institutional Review Board of Wayne State University. Written informed consent was obtained from all participants before examination.

\section{Fetal resting-state $f M R I$}

fMRI acquisition. MRI was examined using a Siemens Verio $70 \mathrm{~cm}$ openbore 3T MR system with a $550 \mathrm{~g}$ abdominal 4-channel Siemens Flex Coil. Echoplanar imaging BOLD parameters included: TR/TE, 2000/30 ms, 360 axial frames, matrix size $96 \times 96 \times 25$, no slice gap, voxel size $3.4 \times$ $3.4 \times 4 \mathrm{~mm}^{3}, 80^{\circ}$ flip angle, repeated twice. Fetal MRI data, subject demographics, and data variables are available at https://nda.nih.gov/ edit_collection.html?id=2434.

Functional data processing. Subsamples of these data have been published before and preprocessing of fetal resting state fMRI followed previously reported methods (Thomason et al., 2013; van den Heuvel et al., 2018). In brief, periods, or epochs, of fetal quiescence were selected using FSL image view (RRID:SCR_002823), and high motion frames were removed manually. Minimum length of a selected epoch was $20 \mathrm{~s}$ (10 frames) of low motion ( $<2 \mathrm{~mm}$ translation, $<3^{\circ}$ rotation $)$. Cases were required to have a minimum of $4 \mathrm{~min}$ of low movement resting state fMRI, resulting in the average of 168 frames $(S D=57)$ across the group. Next, for each dataset, a fetal brain mask was manually drawn around the fetal brain, separately for each low-motion epoch from a single representative image within the epoch using BrainSuite (RRID:SCR_006623). This subject-specific brain mask was used to extract the brain from the maternal compartment for the corresponding epoch. Following this, segmented fetal brains were manually reoriented into standard A-P, R-L, and I-S orientation, with the origin set to the middle of the brain. The quality of reorientation was visually assessed by expert raters by manually checking the alignment of the interhemispheric space with the vertical crosshair for transverse and coronal orientations using Statistical Parametric Mapping software (SPM8; RRID:SCR_007037; Ashburner et al., 2012) implemented in MATLAB. Reoriented, re-centered data epochs were then individually realigned and normalized in SPM. Two normalization approaches were tested to evaluate potential influence of preprocessing steps on study outcomes. Epochs were normalized to a 32-week infant brain template (Serag et al., 2012), representing the group mean, and to age-specific templates; age-specific fetal brain atlas connectome reconstruction. Normalization settings were set to standard settings in SPM, regularization settings were set to the average sized template. All normalized images from each segment were then concatenated into one run, realignment was performed on the concatenated time series to address potential epoch misalignment, and, finally spatial smoothing was performed with a $4 \mathrm{~mm}$ FWHM Gaussian kernel. Connectivity correlation analysis was performed in CONN software (Connectivity Toolbox v14n; RRID:SCR_009550) implemented in MATLAB. For each subject, the 32-week brain template was used as an anatomical reference image and ROIs were selected using FreeSurfer's Desikan Killiany atlas (see fetal connectome reconstruction). The following parameters were used in subject level processing: linear de-trending, bandpass filtering at $0.008-$ $0.09 \mathrm{~Hz}$., and no de-spiking. Nuisance regression included a CompCor of five principal components extracted from white matter and CSF masks, 
fetal MRI

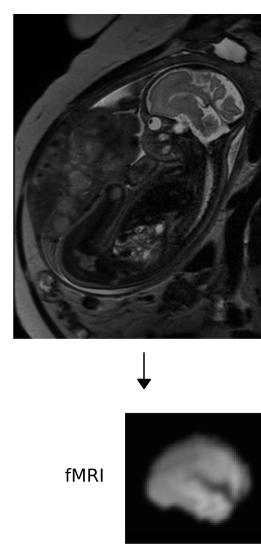

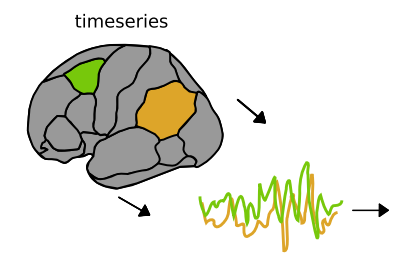

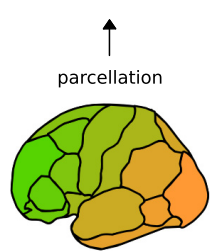

functional connectivity matrix

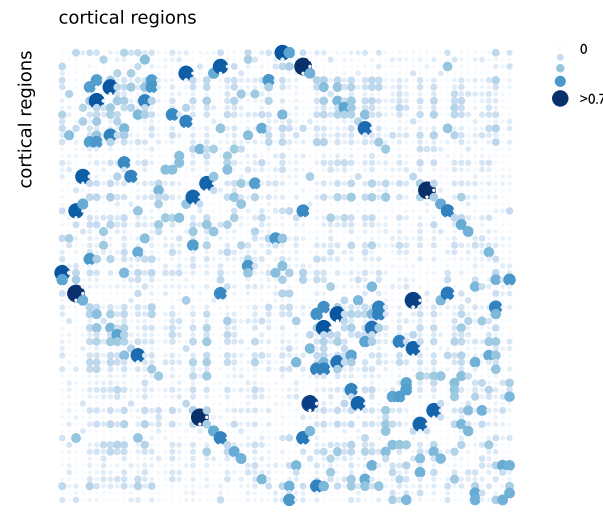

Figure 1. Fetal functional connectome reconstruction. A total of 105 healthy pregnant women underwent MRI examination of the fetal brain, including $T 2$ and fMRI sequences (top left $T 2$ image, courtesy of Wilhelmina Children's Hospital, Utrecht, the Netherlands). Preprocessed resting state fMRI images of 105 fetuses were parcellated using a manually annotated Desikan-Killiany atlas. Subject-level time series from all voxels within a region were averaged and correlated to all other regions of the atlas. This resulted into a FC matrix of 66 cortical nodes, preserving only positive associations (correlation coefficient between 0 and 1 ) in this visual representation.

and also six motion parameters produced by SPM during concatenated segment realignment. White matter and CSF probability masks corresponded to the anatomical reference images generated by Serag et al., 2012) used in the preprocessing steps as described.

\section{Functional connectome reconstruction}

Brain regions were selected using cortical regions of the FreeSurfer's Desikan Killiany atlas reconstructed (including manual fine-tuning in FreeSurfer; RRID:SCR_001847) onto the fetal brain template (Fig. 1). A surrogate $\mathrm{T} 1$ image was created from the $\mathrm{T} 2$ template image by flipping the image contrast and used as input for the pipeline of FreeSurfer. The cortex, subcortex, and ribbon labels were visually inspected and manually fine-tuned using tksurfer. Using the new label files, Freesurfer's mris_ca_label and mri_aparc2aseg were computed, resulting in a fetal parcellation of the cortical mantle of 68 nodes ( 34 regions in each hemisphere). The reconstructed atlas on the fetal brain and cortical ribbon were visually inspected and evaluated by the research team. Voxels of the left and right isthmus cingulate cortex were excluded from further analysis, because inaccurate segmentation of these areas on the 32-week brain template was observed, resulting in 66 regions taken forward for further analysis. FC between brain regions was computed by means of correlation analysis between the recorded regional BOLD time-series, followed by $z$-score transformation. Negative correlations derived from functional resting-state fMRI recordings remain a topic of investigation in the field and were set to zero to reduce contributions of low or potentially spurious cortical interactions. No threshold was used for the modularity analysis to determine resting-state networks (van den Heuvel et al., 2017). A group-averaged fetal connectivity graph was computed across all fetal brains by taking the (nonzero) average of the FC value of all pairs of nodes across all subjects. Connections present in $60 \%$ or more of the total group (Fig. 1) were included in the group connectivity matrix.

\section{Age-specific fetal brain atlas connectome reconstruction}

A second method of functional data processing was performed. Instead of normalizing individual fMRI data to a commonly used single 32-week infant brain template, we examined the use of multiple age-specific brain templates. For this we used an online resource for fetal brain templates and their corresponding atlas and tissue (e.g., developing white matter, CSF) segmentations (CRL Unbiased and Deformable Spatiotemporal Atlas of the Fetal Brain; RRID:SCR_014176; Gholipour et al., 2017). We normalized each fetal fMRI data to a template of the nearest gestational week (which are included in the DSA of 21-38 weeks, with increments of 1 week) using standard settings in SPM8. Visual inspection revealed a total of 90 of 105 subjects, which could be successfully normalized to their age-specific brain template and atlas. The 15 subjects that did not properly normalize to their matching template and datasets were excluded from further analysis. Further preprocessing included the same steps implemented with the Desikan Killiany atlas, comprising spatially smoothing, linear de-trending and nuisance regression using CompCor analysis including five principal components extracted from the agespecific tissue images' white matter and CSF mask, and motion regression. Brain regions for the connectome analysis were selected using cortical regions of the fetal brain atlas (Gholipour et al., 2017) providing a consistent set of 78 regions identified across the fetal brain templates (39 regions in each hemisphere) and FC between brain regions was computed using correlation analysis. All negative correlations were set to zero to reduce contributions of low or potentially spurious cortical interactions. No threshold was used for the modularity analysis.

\section{Network metrics}

Network metrics were computed using the weighted group-averaged FC graph, with metrics computed using both the group-averaged and individual weighted FC graphs of each of the fetal subjects separately. Following prior work, network metrics included calculation of the clustering coefficient, characteristic path length, small world index, rich-club index, and modularity (Rubinov and Sporns, 2010; van den Heuvel and Sporns, 2011). Permutation testing was used to compare fetal FC patterns against 1000 random networks preserving the degree distribution (Maslov and Sneppen, 2002). Normalized network metrics were obtained by taking the ratio of the actual graph metrics and graph metrics observed in the random networks. All results were validated for motion artifacts by computing the network metrics on a subsample of the dataset selecting 52 subjects with most fMRI frames (i.e., 50\% with lowest movement). Clustering coefficient was computed as the average clustering-coefficient $(\mathrm{C} i)$ over all nodes $(i)$ in the network, with $\mathrm{C} i$ describing the ratio between the number of connections between node $i$ and its neighbor-nodes and the total number of possible connections with neighbors. Characteristic path length was computed by the average minimal travel distance (i.e., shortest paths, $L i$ ) between all nodes in the network. Small-world index was computed as the ratio of normalized global clustering coefficient and normalized characteristic path length based on the ratio between $C$ and characteristic path length $(L)$ and the clustering and path length of a set of 1000 random networks $\left(C_{\text {random }}\right.$ and $\left.L_{\text {random }}\right)$. A small-world index $>1$ was used as an indication of a small-world organization of the network.

Rich-club organization of a network describes the above random level of connectivity between the high-strength nodes of the network, with the rich-club describing a subset of nodes more densely interconnected than expected based on their degree alone (Colizza et al., 2006; Opsahl et al., 2008; van den Heuvel and Sporns, 2011). A functional weighted rich-club coefficient $\left[\varphi^{\mathrm{w}}(k)\right]$ was computed as the ratio between the total sum of edge weights that connect nodes of strength $>k$ and the total sum of the strength of the whole network (van den Heuvel and Sporns, 2011). The 
$\varphi^{\mathrm{w}}(k)$ was compared with $\varphi_{\text {random }}^{\mathrm{w}}(k)$ of a set of 1000 randomly rewired networks keeping the same degree distribution (Maslov and Sneppen, 2002), with $\varphi_{\text {norm }}^{\mathrm{w}}(k)$ the ratio between $\varphi^{\mathrm{w}}(k)$ and $\varphi_{\text {random }}^{\mathrm{w}}(k)$. A network is argued to display a rich-club organization when $\varphi(k)>\varphi_{\text {random }}^{\mathrm{w}}(k)$, or equivalent $\varphi_{\text {norm }}^{\mathrm{w}}(k)>1$ (Colizza et al., 2006; van den Heuvel and Sporns, 2011). A $p$ value was assigned to $\varphi^{\mathrm{w}}(k)$ as the percentage of the nulldistribution that exceeded the value of $\varphi^{\mathrm{w}}(k)$. As an alternative definition of centrality, we also examined betweenness centrality, as the fraction of all weighted shortest paths in the network that contain a given node (Fransson et al., 2011; Ball et al., 2014). In this study, the top 15\% nodes with the highest strength or largest values for betweenness centrality were included as central nodes (van den Heuvel and Sporns, 2011).

Community structure of the fetal functional brain network was examined by means of decomposing the network into functional resting-state networks, or modules, determined using Louvain's community detection algorithm (Rubinov and Sporns, 2010). Louvain's community detection algorithm was used on the unthresholded fetal graph, allowing the preservation of both negatively and positively correlated brain regions. Modularity $(Q)$ was computed to quantify the degree to which the network can be subdivided in delineated modules.

\section{Adult connectome used for comparison}

Preprocessed adult fMRI data $(N=42 ; 26$ males $)$ were taken from a previously used dataset (van den Heuvel et al., 2015), including FC matrices and an average participant age of 29 years $(\mathrm{SD}=8)$. The adult $\mathrm{FC}$ matrices were constructed using the Desikan-Killiany atlas to match the 66 regions of the fetal atlas (i.e., 68 minus bilateral isthmus cingulate cortices), allowing for a direct comparison of network structure between the fetal and adult brain. The adult group-averaged graph was computed using similar procedures as constructing the fetal graph, with additional specifications described by van den Heuvel et al. (2015).

\section{Statistical evaluation}

Comparison of the fetal and adult functional connectome. Overlap of the fetal and adult connectome was determined by means of the Mantel test (Mantel, 1967; Scholtens et al., 2014), comparing the level of overlap between the binary group-averaged fetal and adult matrices (threshold = 0 ). Mantel comparison included the computation of a distance matrix, with cell Entry 1 corresponding to overlapping values across the fetal and neonatal matrix and Entry 0 to non-corresponding entries between the fetal and adult group matrix, with the level of overlap taken as the density of the distance matrix, ranging from no overlap (0) to complete overlap (1). Permutation testing with 1000 random networks involved randomizing the binary fetal and adult matrix while preserving degree sequence. Permutation testing was used to assign a $p$ value to the level of overlap between the fetal and adult connectome. Weighted overlap was additionally computed as the level of partial correlation between the two weighted group-averaged graphs across the set of connections (excluding 0 elements) observed in both the fetal and adult connectome. We controlled for Euclidian distance between brain regions, to confirm that the effect of higher connectivity between spatially close regions was not the driven force of the measured overlap, by means of partial correlation analysis.

Comparison of fetal and adult modular organization. Overlap in adult and fetal modular organization was examined using the Rand index (Rand, 1971), taken a pairwise comparison of the number of node pairs that are classified in the same or different modules across the module assignments of the two compared matrices (Karrer et al., 2008), with a Rand index of 0 indicating no overlap and an index of 1 indicating complete overlap. The modular assignments of the nodes of both the adult and fetus were randomized across 1000 permutations, and the Rand index in the random situations was computed as a null-distribution. The resulting null distribution of random overlap effects was used to assign a $p$ value based on the percentage of observations in the random condition exceeding the original value. Within module connectivity, strength was computed for each node ( $i$ ) as the total number, or degree, of connection, multiplied with their weights, or strength, attached to all nodes in one module.

\section{Evaluation of the robustness of the data}

Given the sensitivity of resting state data to motion and complexity of analyzing fMRI in a fetal cohort, multiple control analyses were performed to estimate the variance within and between groups and thereby evaluate the robustness of findings. As a control condition, we examined fetal group network metrics for the subgroup of low-motion participants separately (i.e., $50 \%$ of the population with the lowest movement during fMRI acquisition). Additionally, control analyses were performed on the robustness of the fetal group connectome. Fetal subjects were 1000 times split into two randomly chosen equal-sized groups and new group matrices were computed. Comparing the overlap of different matrices, correlation analyses with and without controlling for the adult connectome were performed on two random fetal group matrices. Third, we addressed potential concerns regarding possible influence of the whitematter regressor on the fMRI signal. Performing white-matter regression on fMRI is a normal strategy in adult subjects. However, the developing myelination and changing intensity of the white matter in fetal subjects may influence the utility of this regressor at different fetal ages. To address this, all network metrics and the association with the adult connectome were recomputed on fetal matrices computed excluding white matter as a regressor, and results were compared across approaches.

\section{Results}

\section{Network metrics}

Group-averaged fetal connectome (Fig. 1) showed a density of $42 \%$, significantly higher clustering (1.20 times higher than one would expect in 1000 random networks; $p<0.001$ ), longer path length (1.14 times longer than 1000 random networks; $p<$ 0.001 ), and a small-world index $>1$ (1.05 times higher than 1000 random networks; $p<0.001)$. Group-averaged fetal connectome reconstructed from the age-matched fetal brain atlas (Gholipour et al., 2017) similarly showed significantly higher clustering (2.62 times higher than one would expect in 1000 random networks; $p<0.001)$, longer path length (1.13 times longer than 1000 random networks; $p<0.001)$, and a small-world index $>1$ (1.97 times higher than 1000 random networks; $p<0.001$ ).

\section{Modular organization}

The fetal connectome showed a clear modular organization $(Q=$ 0.28 ) describing four functional modules. These four functional modules included: (1) an occipital and parietal visuosomatosensory module, with left and right cuneus, lingual, paracentral, pericalcarine, posterior cingulate, precuneus, and superior parietal cortical regions; 2) a midline prefrontal-temporal-insular module with left and right bankssts (i.e., banks of the superior temporal sulcus), caudal middle frontal, rostral anterior cingulate, superior temporal, transverse temporal, insula, right temporal pole, and left medial orbitofrontal cortical regions; 3) a temporal module with left and right entorhinal, fusiform, inferior temporal, parahippocampal, and left temporal pole cortical regions; and 4) an extensive lateral and midline frontal module including left and right caudal anterior cingulate, inferior parietal, lateral occipital, lateral orbitofrontal, middle temporal, parsopercularis, parsorbitalis, parstriangularis, postcentral, precentral, rostral middle frontal, superior frontal, supramarginal, frontal pole, and right medial orbitofrontal cortical regions (Fig. 2a).

The fetal connectome reconstructed from the age-matched fetal brain atlas showed a high modular organization $(Q=0.53)$ revealing two additional functional modules, with this higher number of modules compared with fixed 32-week atlas analysis likely related to the larger amount of regions in the age-matched fetal brain atlas. The age-matched fetal brain atlas revealed: (1) a visual module, with left and right superior, middle, inferior occipital cortices, the left and right precuneus, cuneus, calcerine, and posterior cingulum. (2) A somatosensory motor module, 
with left and right superior frontal, superior medial frontal, supplementary motor, paracentral, and mid-cingulum and left precentral, midfrontal, postcentral, superior, and inferior parietal, supramarginal, and angular cortices. (3) A temporal and orbitofrontal module with left and right superior, mid, medial, and inferior orbitofrontal cortices, left and right olfactory, rectus, parahippocampal, fusiform, mid-temporal pole, inferior temporal cortices. (4) A right-oriented midfrontal-temporal-insular-parietal module, with right inferior frontal and rolandic operculum, frontal inferior triangularis, anterior cingulum, mid, superior temporal cortices and superior temporal pole, hescl, insular, precentral, frontal, postcentral, superior, and inferior parietal, supramaginal, and angular cortices. (5) A left oriented frontal-temporalinsular module, with left inferior frontal and rolandic operculum, frontal inferior triangularis, anterior cingulum, mid, superior temporal cortices and superior temporal pole, hescl and insular cortices, and right anterior cingulum.

\section{Hub organization}

The fetal connectome showed a significant rich-club organization for the range of $22 \leq k \leq 38$ [ $\varphi_{\text {norm }}^{\mathrm{w}}(k)>1, p<0.001$, 1000 random networks; Fig. $2 b]$. The strongest functionally connected nodes of the fetal brain included: bilateral orbitofrontal, postcentral and middle temporal cortical regions, left entorhinal regions as well as the right parsorbitalis, medial orbitofrontal regions and fusiform area. Based on weighted betweenness centrality, the top $15 \%$ highest betweenness centrality nodes in the fetal brain included: bilateral lateral occipital, postcentral, right superior frontal, postcentral, fusiform and left insular, middle temporal, lateral, and medial orbitofrontal cortical regions. Richclub index of the Gholipour atlas similarly revealed levels $>1$ $(p<0.001)$. Based on the FC strength of the group averaged matrix, the top $15 \%$ highest strength nodes included: homologous parts of the precentral, precuneus, postcentral, right insula, and left superior parietal and superior (orbito-) frontal regions. Based on the weighted betweenness centrality, the top 15\% densely connected nodes in the fetal cortex included: homologous parts of the superior orbitofrontal cortex, the insula, left mid orbitofrontal cortex, and right regions of the olfactory bulb, angular, mid temporal, rectus, supplementary motor regions, and medial superior frontal cortex. Combining these findings, our results suggest that the strongest functionally connected regions in the fetal brain are predominantly located in the temporal cortical regions and around the midline of the brain including especially frontal, postcentral, and insular cortices.

\section{Overlap between fetal and adult connectome}

We continued our analysis by examining the overlap of global and modular organization of the fetal and adult cerebral functional connectome. First, a significant overlap of $61.66 \%$ (Mantel test, $p<0.001$ ) was found between the fetal and adult connectome. The strength of all connections observed in both the fetal and adult cortex showed a significant positive association $(r=$ $0.389, p<0.001)$. The association remained significant when further controlling for Euclidian distance $(r=0.299, p<0.001)$. These data suggest early presence of an adult-like distribution of FC in the human intrauterine connectome. Second, the adult group-averaged matrix revealed five distinct functional modules describing a temporal, default-mode, visual, frontomedial, and motor functional network, in contrast to the four modules that were observed in the fetal brain. Side-by-side comparison of the modularity-matrices shows that the fetal connectome has adultlike functional networks components, although they are in an immature state (Fig. 3). Subsequently, fetal and adult group graphs revealed a high modularity overlap (Rand index: 0.730 , $p<0.001)$.

Strong FC overlap between fetal and adult within-module connectivity was found in the visual module $(r=0.597, p<$ $0.001)$, temporal module $(0.727, p<0.001)$, default mode module $(r=0.608, p<0.001)$, motor module $(0.324, p<0.001)$, whereas lower overlap was found in the frontomedial module $(r=0.181 . p<0.001)$, indicating an early presence of mature networks for four of five data-derived modules.

\section{Robustness analyses}

Motion analysis

High clustering, short path length and small-worldness organization were significantly replicated in the subset of 52 low-motion participants (i.e., $50 \%$ of the population with lowest movement). Eight of 10 most functionally connected areas (highest strength) were replicated. The areas that did not replicate as highly connected areas in the 52 low-motion participants were left postcentral and middle temporal gyrus, which were replaced by left fusiform and left insula. Similarly, 8 of 10 most centrally connected regions were 

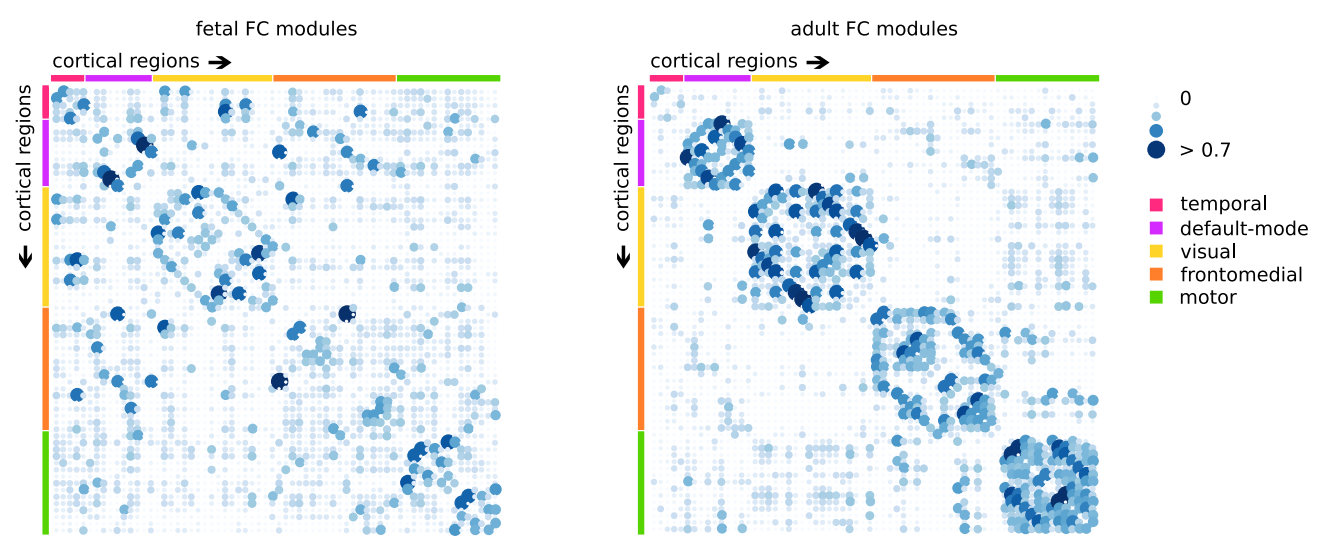

Figure 3. Comparison of group averaged fetal and adult functional connectome modules. Illustration of the modular organization modules of the fetal (left) and adult (right) connectivity matrices. Blue dots represent positive correlations between nodes; the upper $10 \%$ of the highest connections are shown. Both matrices display the adult modular organization, revealing a temporal, default-mode, visual, frontomedial, and motor network.

replicated in the low-motion subgroup. Areas that did not replicate were left middle and medial temporal cortical regions, which were replaced by right insula and left superior frontal cortex. Furthermore, we observed that the modules that were identified were largely replicated when repeating this analysis in the subset of low motion subjects; the only variation observed was expansion of the temporal module to cover a larger extent of the temporal cortex.

\section{Robustness of the fetal group connectome}

Splitting the fetal subjects into two random groups, significant associations were found comparing both group matrices (all 1000 correlations between $r=0.935$ and $r=0.975, p<0.001)$, and when controlled for the adult matrix, the correlation remained significant $(r=0.811$ and $r=0.905, p<0.001)$. Correlation analysis between different fetal subgroups revealed strong positive significant within-module connectivity correlations (all $r$ values $>0.9, p<0.001$ ) for all four modules.

\section{White-matter regressor analysis}

Robustness analysis further revealed a significant association $(r=$ $0.722, p<0.001$ ) between the mean group matrices of the fetal group computed with and without global white-matter correction. A higher density of the group matrix was found $(94 \%, t=0$; $58 \%, t=0.1 ; 21 \%, t=0.2 ; 10 \%, t=0.3)$ as a direct consequence of excluding white matter as a regressor to calculate subject FC matrices. Without global correction of white matter on the fMRI signal, the use of absolute thresholding (i.e., including correlations above a threshold $t>0.1$ ) was thus needed to replicate similar network densities, replicating high clustering, short path length and small-worldness organization in the fetal group matrix reconstructed from fetal FC analysis without white-matter regression. The overlap of the strongest functionally connected nodes ranged between $20(t=0.3), 30(t=0.1)$ and $40 \%(t=0.2)$ and central nodes ranged between $50(t=0.1), 50(t=0.3)$, and $70 \%(t=0.2)$, varying threshold $t$. Four functional modules were again observed, with the original fetal group graphs, with and without white-matter regression, showing a high level of modularity overlap (Rand index: $0.675, p<0.001$ ). Significant associations were revealed comparing the alternative fetal and adult matrices using different thresholds $(t=0.1, r=0.504, p<0.001$; $t=0.2, r=0.476, p<0.001$ and $t=0.3, r=0.402, p<0.001)$, remaining significant when controlled for Euclidian distance (respectively, $r=0.365, p<0.001 ; r=0.429, p<0.001$ and $r=$ $0.465, p<0.001)$.

\section{Discussion}

The present study confirms the presence of a functional brain connectomic blueprint in the second and third trimester of pregnancy in utero. The results of this large fetal functional restingstate MRI cohort show the early presence of fundamental properties of the neural connectome, including a functional small-world, modular, and rich-club architecture. Our findings suggest the presence of both primary motor and sensory networks as well as the first signs of higher order networks that are pruned for laterlife cognition. We observe a high overlap between adult and fetal functional organization of the cortex, indicating that mature functional dynamics have their origin before birth. Extending previous observations of (premature) neonatal resting-state networks (Fransson et al., 2009, 2011; Gao et al., 2009, 2015; Doria et al., 2010; Smyser et al., 2010; Schöpf et al., 2012; Thomason et al., 2013; De Asis-Cruz et al., 2015; He and Parikh, 2016) our results complement the notion of the early presence of a blueprint of the formation of resting-state networks in the healthy second to third trimester fetal brain, now shown for the fetal developing brain. The connectome emerges and is detectable with fetal fMRI in the second trimester of pregnancy, when FC patterns show first signs of organization into a complex network of communicating brain regions.

MRI studies have shown a small-world type of network organization with functional and structural rich-club hubs, increasing global efficiency, as well as structural and functional architectural coupling in the developing (preterm) neonatal brain (Fransson et al., 2011; De Asis-Cruz et al., 2015; van den Heuvel et al., 2015; Scheinost et al., 2016; Cao et al., 2017). Major white-matter pathways associated with the development of the structural rich club in preterm neonates are established before the third trimester of pregnancy (Ball et al., 2014; van den Heuvel et al., 2015). Our findings show that the most densely functionally connected areas (which one could refer to as "functional hubs") in the fetal cortex are predominantly confined to temporal and midline cortical regions of the insular and frontal lobes, as well as the primary somatosensory regions. Functional MRI and EEG studies analyzing functional hub organization of the neonatal brain show similar findings, with medial temporal, fusiform, and primary somatosensory regions commonly identified as densely connected hub areas in the early developing brain (Gao et al., 2009; Fransson et al., 2011; De Asis-Cruz et al., 2015; Arichi et al., 2017; van den Heuvel et al., 2018), as well as inferior frontal (Fransson 
et al., 2011) and orbitofrontal regions (e.g., as part of the rolandic operculum in the study by De Asis-Cruz et al., 2015), and insular cortices (Gao et al., 2011; De Asis-Cruz et al., 2015; Arichi et al., 2017). Combining fetal and preterm neonatal findings, we hypothesize that fetal hubs fulfill an important role in coordinating activity, predominantly linked to brain functions that are associated with primary functions.

Our results show high overlap of fetal and adult resting-state networks that are pruned to coordinate motor, visual, auditory, and some cognitive functions, whereas the frontomedial network that is especially associated with higher cognitive functions, shows a less strong association. These findings are in line with previous fMRI findings in preterm neonates showing that prototypes of resting-state networks are developed before or around term birth (Gao et al., 2009; Doria et al., 2010; Smyser et al., 2010; Schöpf et al., 2012; Thomason et al., 2013, 2015; van den Heuvel et al., 2015). In contrast, the frontoparietal network is still fragmented around birth and during the first year of life (Fransson et al., 2009; Gao et al., 2015; He and Parikh, 2016). Disruptions of the developing somatomotor and frontoparietal functional networks around term equivalent age has been recently linked to motor impairments in the first 4-8 months of life (Linke et al., 2018). Combining these findings we observe that local development of the functional architecture of the brain before birth is dynamic and develops in a sequential primary to higher cognitive fashion (Gao et al., 2009; Doria et al., 2010; Smyser et al., 2010; Schöpf et al., 2012; Thomason et al., 2013, 2015; van den Heuvel et al., 2015).

The participant group and the nature of the imaging technique include several limitations. The following points should thus be taken into consideration when interpreting our presented findings. First, we included fetuses that were examined at a wide range of ages, which enabled cross-sectional developmental assessment, but also resulted in averaging across a large age range when making group level comparisons. Similar to former studies, we limited this effect through spatial normalization and realignment of fMRI volumes to a standard average 32-week gestation template brain (Serag et al., 2012), but larger statistical variation in the fetal group is to be expected. We therefore validated our results by including a second normalization method using agespecific brain templates (Gholipour et al., 2017). Second, another major consideration is that imaging the fetal brain in vivo is influenced by maternal respiration, high movement, and the changing position of the fetus (Ferrazzi et al., 2014). Although post-processing techniques within this study include several steps to reduce motion artifacts such as regression out of motion parameters and manual scrubbing, noisy artifacts may remain in the study data. Noisy artifacts can lead to a reduction of long-range connectivity between distant regions, and as well to an increase in connectivity between proximal brain regions (Power et al., 2012; Van Dijk et al., 2012). Despite the challenging analysis, our findings suggest that motion and other artifacts are not the main driving factors of our presented results. Furthermore, changing white-matter intensities with time due to myelination maturation may influence the fMRI signal. Including white-matter regression brings the opportunity to take out signals from within the brain that capture both signals arising from the white matter and potential spurious contributions of noise. Our results show that comparable results are found including and excluding the white-matter signal as a regressor in the FC analysis, but dissimilarities can be pointed out as well because of the difference in global correction. Furthermore, the high overlap between resting-state FC distributions of the fetal and healthy adult brain indicates high enough accuracy in measurement of prenatal FC. Third, in the discussion, we compare our fetal fMRI findings with reports on preterm neonatal brain organization. The brain of preterm infants without overt brain injury provides insight into the same time period, and properties of global functional dynamics measured in utero appear to largely overlap with those observed in preterm infants (Ferrazzi et al., 2014). It should be noted that the preterm neonatal brain is not a perfect surrogate for typical processes of brain circuitry development, because premature birth, absence of neuroprotective elements, neonatal intensive care unit environment, and medical interventions can be harmful for the developing brain (Karmel et al., 2010; Brummelte et al., 2012; Smyser et al., 2012; Groppo et al., 2014; Pritchard et al., 2014). Additionally, early exposure to the extra-uterine environment may accelerate brain function because of introduction of sensory stimuli (Klebermass et al., 2006). Fetal fMRI-based network analysis is therefore crucial in understanding healthy connectome development. Results presented here show that the fetal period is important for the development of specialized functional domains in an economic and integrative network before birth, suggesting that the prenatal period is important to point out vulnerable windows for developing later life brain function deficits. It does not show a complete model yet, but our findings do provide new insights into foundational aspects of fetal brain development.

Our findings suggest that fetal MRI provides the possibility to map the blueprint of functional connectome organization during the earliest phases of brain development. The rapid nature of neurodevelopmental processes during pregnancy may render the human brain at elevated risk for connectome alterations. Fetal MRI currently serves as a clinical tool for the early detection of brain abnormalities, such as agenesis of the corpus callosum and other cerebral anomalies (Volpe et al., 2006), or delayed brain maturation in fetuses with severe congenital heart disease (Claessens et al., 2019). Considering that organizational properties of brain networks associated with later life motoric and cognitive functioning are already apparent in the fetal brain across $20-40$ weeks of gestation, it may in the future be possible to complement clinical tools to detect early signs, or origins, of psychopathology using fetal fMRI. It would be interesting to search deeper into possible risk factors, such as maternal stress, alcohol and drugs intake, prematurity, fetal growth restriction, premature birth, or cardiac dysfunction, that could influence healthy fetal brain circuitry, with lasting implication for individual trajectories of human development. Further, it will be important to link early functional dynamics to structural patterns. Few studies have examined functional or structural differences between healthy fetal and preterm neonatal development (Bouyssi-Kobar et al., 2016; Thomason et al., 2017) and they have shown that both altered FC and cortical folding differences can already discernable in this period.

\section{References}

Anderson AL, Thomason ME (2013) Functional plasticity before the cradle: a review of neural functional imaging in the human fetus. Neurosci Biobehav Rev 37:2220-2232.

Arichi T, Whitehead K, Barone G, Pressler R, Padormo F, Edwards AD, Fabrizi L (2017) Localization of spontaneous bursting neuronal activity in the preterm human brain with simultaneous EEG-fMRI. eLife 6:e27814.

Ashburner J, Barnes G, Chen C, Daunizeau J, Flandin G, Friston K, Gitelman D, Kiebel S, Kilner J, Litvak V (2012) SPM8 manual. London UK: Functional Imaging Laboratory, Institute of Neurology.

Ball G, Aljabar P, Zebari S, Tusor N, Arichi T, Merchant N, Robinson EC, Ogundipe E, Rueckert D, Edwards AD, Counsell SJ (2014) Rich-club 
organization of the newborn human brain. Proc Natl Acad Sci U S A 111:7456-7461.

Bouyssi-Kobar M, du Plessis AJ, McCarter R, Brossard-Racine M, Murnick J, Tinkleman L, Robertson RL, Limperopoulos C (2016) Third trimester brain growth in preterm infants compared with in utero healthy fetuses. Pediatrics 138:e20161640.

Brummelte S, Grunau RE, Chau V, Poskitt KJ, Brant R, Vinall J, Gover A, Synnes AR, Miller SP (2012) Procedural pain and brain development in premature newborns. Ann Neurol 71:385-396.

Cao M, He Y, Dai Z, Liao X, Jeon T, Ouyang M, Chalak L, Bi Y, Rollins N, Dong Q, Huang H (2017) Early development of functional network segregation revealed by connectomic analysis of the preterm human brain. Cereb Cortex 27:1949-1963.

Claessens N, Khalili N, Isgum I, ter Heide H, Steenhuis T, Turk E, Steenhuis TJ, Jansen N, de Vries L, Breur J, de Heus R, Benders MJ (2019) Brain and CSF volumes in fetuses and neonates with antenatal diagnosis of critical congenital heart disease: a longitudinal MRI study. AJNR Am J Neuroradiol 40:885-891.

Colizza V, Flammini A, Serrano MA, Vespignani A (2006) Detecting richclub ordering in complex networks. Nat Physics 2:110-115.

Collin G, van den Heuvel MP (2013) The ontogeny of the human connectome development and dynamic changes of brain connectivity across the life span. Neuroscientist 19:616-628.

De Asis-Cruz J, Bouyssi-Kobar M, Evangelou I, Vezina G, Limperopoulos C (2015) Functional properties of resting state networks in healthy fullterm newborns. Sci Rep 5:17755.

de Water E, Proal E, Wang V, Medina SM, Schnaas L, Téllez-Rojo MM, Wright RO, Tang CY, Horton MK (2018) Prenatal manganese exposure and intrinsic functional connectivity of emotional brain areas in children. Neurotoxicology 64:85-93.

Doria V, Beckmann CF, Arichi T, Merchant N, Groppo M, Turkheimer FE, Counsell SJ, Murgasova M, Aljabar P, Nunes RG, Larkman DJ, Rees G, Edwards AD (2010) Emergence of resting state networks in the preterm human brain. Proc Natl Acad Sci U S A 107:20015-20020.

Fair DA, Cohen AL, Power JD, Dosenbach NU, Church JA, Miezin FM, Schlaggar BL, Petersen SE (2009) Functional brain networks develop from a "local to distributed" organization. PLoS Comput Biol 5:e1000381.

Ferrazzi G, Kuklisova Murgasova M, Arichi T, Malamateniou C, Fox MJ, Makropoulos A, Allsop J, Rutherford M, Malik S, Aljabar P, Hajnal JV (2014) Resting state fMRI in the moving fetus: a robust framework for motion, bias field and spin history correction. Neuroimage 101:555-568.

Fransson P, Skiöld B, Engström M, Hallberg B, Mosskin M, Åden U, Lagercrantz H, Blennow M (2009) Spontaneous brain activity in the newborn brain during natural sleep: an fMRI study in infants born at full term. Pediatr Res 66:301-305.

Fransson P, Åden U, Blennow M, Lagercrantz H (2011) The functional architecture of the infant brain as revealed by resting-state fMRI. Cereb Cortex 21:145-154.

Gao W, Zhu H, Giovanello KS, Smith JK, Shen D, Gilmore JH, Lin W (2009) Evidence on the emergence of the brain's default network from 2-weekold to 2-year-old healthy pediatric subjects. Proc Natl Acad Sci U S A 106:6790-6795.

Gao W, Gilmore JH, Giovanello KS, Smith JK, Shen D, Zhu H, Lin W (2011) Temporal and spatial evolution of brain network topology during the first two years of life. PLoS One 6:e25278.

Gao W, Alcauter S, Smith JK, Gilmore JH, Lin W (2015) Development of human brain cortical network architecture during infancy. Brain Struct Funct 220:1173-1186.

Gholipour A, Rollins CK, Velasco-Annis C, Ouaalam A, Akhondi-Asl A, Afacan O, Ortinau CM, Clancy S, Limperopoulos C, Yang E, Estroff JA, Warfield SK (2017) A normative spatiotemporal MRI atlas of the fetal brain for automatic segmentation and analysis of early brain growth. Sci Rep 7:476.

Grewen K, Salzwedel AP, Gao W (2015) Functional connectivity disruption in neonates with prenatal marijuana exposure. Front Hum Neurosci 9:601.

Groppo M, Ricci D, Bassi L, Merchant N, Doria V, Arichi T, Allsop JM, Ramenghi L, Fox MJ, Cowan FM, Counsell SJ, Edwards AD (2014) Development of the optic radiations and visual function after premature birth. Cortex 56:30-37.

He L, Parikh NA (2016) Brain functional network connectivity develop- ment in very preterm infants: the first six months. Early Hum Dev 98:29-35.

Hoff GE, Van den Heuvel M, Benders MJ, Kersbergen KJ, De Vries LS (2013) On development of functional brain connectivity in the young brain. Front Hum Neurosci 7:650.

Huttenlocher PR, Dabholkar AS (1997) Regional differences in synaptogenesis in human cerebral cortex. J Comp Neurol 387:167-178.

Infante MA, Moore EM, Bischoff-Grethe A, Tapert SF, Mattson SN, Riley EP (2017) Altered functional connectivity during spatial working memory in children with heavy prenatal alcohol exposure. Alcohol 64:11-21.

Jakab A, Schwartz E, Kasprian G, Gruber GM, Prayer D, Schöpf V, Langs G (2014) Fetal functional imaging portrays heterogeneous development of emerging human brain networks. Front Hum Neurosci 8:852.

Karmel BZ, Gardner JM, Meade LS, Cohen IL, London E, Flory MJ, Lennon EM, Miroshnichenko I, Rabinowitz S, Parab S, Barone A, Harin A (2010) Early medical and behavioral characteristics of NICU infants later classified with ASD. Pediatrics 126:457-467.

Karrer B, Levina E, Newman ME (2008) Robustness of community structure in networks. Phys Rev E Stat Nonlin Soft Matter Phys 77:046119.

Klebermass K, Kuhle S, Olischar M, Rücklinger E, Pollak A, Weninger M (2006) Intra-and extrauterine maturation of amplitude-integrated electroencephalographic activity in preterm infants younger than 30 weeks of gestation. Biol Neonate 89:120-125.

Kostović I, Jovanov-Milošević N (2006) The development of cerebral connections during the first 20-45 weeks' gestation. Semin Fetal Neonatal Med 11:415-422.

Li X, Andres A, Shankar K, Pivik R, Glasier C, Ramakrishnaiah R, Zhang Y, Badger T, Ou X (2016) Differences in brain functional connectivity at resting state in neonates born to healthy obese or normal-weight mothers. Int J Obes 40:1931-1934.

Lin W, Zhu Q, Gao W, Chen Y, Toh CH, Styner M, Gerig G, Smith J, Biswal B, Gilmore J (2008) Functional connectivity MR imaging reveals cortical functional connectivity in the developing brain. Am J Neuroradiol 29:1883-1889.

Linke AC, Wild C, Zubiaurre-Elorza L, Herzmann C, Duffy H, Han VK, Lee DS, Cusack R (2018) Disruption to functional networks in neonates with perinatal brain injury predicts motor skills at 8 months. Neuroimage Clin 18:399-406.

Mantel N (1967) The detection of disease clustering and a generalized regression approach. Cancer Res 27:209-220.

Maslov S, Sneppen K (2002) Specificity and stability in topology of protein networks. Science 296:910-913.

Mrzljak L, Uylings HB, Van Eden CG, Judás M (1991) Neuronal development in human prefrontal cortex in prenatal and postnatal stages. Prog Brain Res 85:185-222.

Opsahl T, Colizza V, Panzarasa P, Ramasco JJ (2008) Prominence and control: the weighted rich-club effect. Phys Rev Lett 101:168702.

Power JD, Fair DA, Schlaggar BL, Petersen SE (2010) The development of human functional brain networks. Neuron 67:735-748.

Power JD, Barnes KA, Snyder AZ, Schlaggar BL, Petersen SE (2012) Spurious but systematic correlations in functional connectivity MRI networks arise from subject motion. Neuroimage 59:2142-2154.

Pritchard VE, Bora S, Austin NC, Levin KJ, Woodward LJ (2014) Identifying very preterm children at educational risk using a school readiness framework. Pediatrics 134:e825-e832.

Rand WM (1971) Objective criteria for the evaluation of clustering methods. J Am Stat Assoc 66:846-850.

Rubinov M, Sporns O (2010) Complex network measures of brain connectivity: uses and interpretations. Neuroimage 52:1059-1069.

Rudolph MD, Graham AM, Feczko E, Miranda-Dominguez O, Rasmussen JM, Nardos R, Entringer S, Wadhwa PD, Buss C, Fair DA (2018) Maternal IL-6 during pregnancy can be estimated from newborn brain connectivity and predicts future working memory in offspring. Nat Neurosci 21:765-772.

Salzwedel AP, Grewen KM, Vachet C, Gerig G, Lin W, Gao W (2015) Prenatal drug exposure affects neonatal brain functional connectivity. J Neurosci 35:5860-5869.

Salzwedel AP, Grewen KM, Goldman BD, Gao W (2016) Thalamocortical functional connectivity and behavioral disruptions in neonates with prenatal cocaine exposure. Neurotoxicol Teratol 56:16-25.

Scheinost D, Kwon SH, Shen X, Lacadie C, Schneider KC, Dai F, Ment LR, 
Constable RT (2016) Preterm birth alters neonatal, functional rich club organization. Brain Struct Funct 221:3211-3222.

Scholtens LH, Schmidt R, de Reus MA, van den Heuvel MP (2014) Linking macroscale graph analytical organization to microscale neuroarchitectonics in the macaque connectome. J Neurosci 34:12192-12205.

Schöpf V, Kasprian G, Brugger P, Prayer D (2012) Watching the fetal brain at "rest". Int J Dev Neurosci 30:11-17.

Schöpf V, Schlegl T, Jakab A, Kasprian G, Woitek R, Prayer D, Langs G (2014) The relationship between eye movement and vision develops before birth. Front Hum Neurosci 8:775.

Serag A, Aljabar P, Ball G, Counsell SJ, Boardman JP, Rutherford MA, Edwards AD, Hajnal JV, Rueckert D (2012) Construction of a consistent high-definition spatio-temporal atlas of the developing brain using adaptive kernel regression. Neuroimage 59:2255-2265.

Sidman RL, Rakic P (1973) Neuronal migration, with special reference to developing human brain: a review. Brain Res 62:1-35.

Smyser CD, Inder TE, Shimony JS, Hill JE, Degnan AJ, Snyder AZ, Neil JJ (2010) Longitudinal analysis of neural network development in preterm infants. Cereb Cortex 20:2852-2862.

Smyser CD, Kidokoro H, Inder TE (2012) Magnetic resonance imaging of the brain at term equivalent age in extremely premature neonates: to scan or not to scan? J Paediatr Child Health 48:794-800.

Thomason ME (2018) Structured spontaneity: building circuits in the human prenatal brain. Trends Neurosci 41:1-3.

Thomason ME, Dassanayake MT, Shen S, Katkuri Y, Alexis M, Anderson AL, Yeo L, Mody S, Hernandez-Andrade E, Hassan SS, Studholme C, Jeong JW, Romero R (2013) Cross-hemispheric functional connectivity in the human fetal brain. Sci Transl Med 5:173ra24.

Thomason ME, Grove LE, Lozon TA Jr, Vila AM, Ye Y, Nye MJ, Manning JH, Pappas A, Hernandez-Andrade E, Yeo L, Mody S, Berman S, Hassan SS, Romero R (2015) Age-related increases in long-range connectivity in fetal functional neural connectivity networks in utero. Dev Cogn Neurosci 11:96-104.

Thomason ME, Scheinost D, Manning JH, Grove LE, Hect J, Marshall N,
Hernandez-Andrade E, Berman S, Pappas A, Yeo L, Hassan SS, Constable RT, Ment LR, Romero R (2017) Weak functional connectivity in the human fetal brain prior to preterm birth. Sci Rep 7:39286.

van den Bergh BR, van den Heuvel MI, Lahti M, Braeken M, de Rooij SR, Entringer S, Hoyer D, Roseboom T, Räikkönen K, King S, Schwab M (2017) Prenatal developmental origins of behavior and mental health: the influence of maternal stress in pregnancy. Neurosci Biobehav Rev. Advance online publication. Retrieved July 28, 2017. doi:10.1016/j. neubiorev.2017.07.003

van den Heuvel MI, Thomason ME (2016) Functional connectivity of the human brain in utero. Trends Cogn Sci 20:931-939.

van den Heuvel MI, Turk E, Manning JH, Hect J, Hernandez-Andrade E, Hassan SS, Romero R, van den Heuvel MP, Thomason ME (2018) Hubs in the human fetal brain network. Dev Cogn Neurosci 30:108-115.

van den Heuvel MP, Sporns O (2011) Rich-club organization of the human connectome. J Neurosci 31:15775-15786.

van den Heuvel MP, Kersbergen KJ, de Reus MA, Keunen K, Kahn RS, Groenendaal F, de Vries LS, Benders MJ (2015) The neonatal connectome during preterm brain development. Cereb Cortex 25:3000-3013.

van den Heuvel MP, de Lange SC, Zalesky A, Seguin C, Yeo BT, Schmidt R (2017) Proportional thresholding in resting-state fMRI functional connectivity networks and consequences for patient-control connectome studies: issues and recommendations. Neuroimage 152:437-449.

Van Dijk KR, Sabuncu MR, Buckner RL (2012) The influence of head motion on intrinsic functional connectivity MRI. Neuroimage 59:431-438.

Vasung L, Huang H, Jovanov-Milošević N, Pletikos M, Mori S, Kostović I (2010) Development of axonal pathways in the human fetal frontolimbic brain: histochemical characterization and diffusion tensor imaging. J Anat 217:400-417.

Volpe P, Paladini D, Resta M, Stanziano A, Salvatore M, Quarantelli M, De Robertis V, Buonadonna AL, Caruso G, Gentile M (2006) Characteristics, associations and outcome of partial agenesis of the corpus callosum in the fetus. Ultrasound Obstet Gynecol 27:509-516. 